STABILITY IN PROBABILITY

BANACH CENTER PUBLICATIONS, VOLUME 90

INSTITUTE OF MATHEMATICS

POLISH ACADEMY OF SCIENCES

WARSZAWA 2010

\title{
ON THE POWER OF AN ASYMPTOTICALLY OPTIMAL TEST FOR THE CASE OF LAPLACE DISTRIBUTION
}

\author{
ROMAN A. KOROLEV and VLADIMIR E. BENING \\ Department of Mathematical Statistics \\ Faculty of Computational Mathematics and Cybernetics \\ Lomonosov Moscow State University \\ Vorobievy gory, 119992, GSP-2, Moscow, Russian Federation \\ E-mail: stochastique@gmail.com,bening@yandex.ru
}

\begin{abstract}
In the paper we prove a formula for the limit of the difference between the power of the asymptotically optimal test and the power of the asymptotically most powerful test for the case of Laplace distribution.
\end{abstract}

1. Introduction. The Laplace distribution was introduced by P. S. Laplace in 1774 as the first law of error which states that the frequency of an error could be expressed as an exponential function of the numerical magnitude of the error, disregarding sign, or equivalently that the logarithm of the frequency of an error (without regard to sign) is a linear function of the error (see [11], pp. 3-13). The second law of error (proposed four years later in 1778) states that the logarithm of the frequency of the error is a quadratic (parabolic) function of the error. The second Laplace law is usually called the normal distribution or the Gauss law. E. B. Wilson noted that there are excellent mathematical reasons for the far greater attention that has been paid to the second law, since it involves the variable $x^{2}$ (if $x$ is the error) and this is subject to all the laws of elementary mathematical analysis, while the first law involving the absolute value of the error $x$ is not an analytic function and presents considerable mathematical difficulty in its manipulation (see [11], pp. 3-13). Due to this non-regularity the first law of error was forgotten for about 150 years, and in 1911 the famous economist and probabilist J. M. Keynes obtained the first law error again from the assumption that the most probable value of the measured quantity is equal to the median of measurements. Later in $1923 \mathrm{E}$. B. Wilson suggested that the frequency we actually meet in everyday work in economics, biometrics, or vital

2010 Mathematics Subject Classification: 62-06, 62F03, 62 F05.

Key words and phrases: power function, Laplace or double exponential distribution. The paper is in final form and no version of it will be published elsewhere. 
statistics often fails to conform closely to the normal distribution, and that Laplace's first law should be considered as a candidate for fitting data in economics and health sciences (see [11], pp. 3-13). Nowadays there are many applications of the Laplace distribution to communications, economics, engineering, and finance (see [11]).

In our paper we employ the theory of testing statistical hypotheses for the case of Laplace distribution using an asymptotic approach. The asymptotic approach is applied when use of the uniformly most powerful test is difficult, or the latter does not exist. In practice the main aim of this approach is to calculate the power loss of the asymptotically optimal test which is closely related to the deficiency of the corresponding test (see [1]). The power loss is obtained as the difference between the power of the asymptotically optimal test and the power of the asymptotically most powerful test.

We consider a problem of testing a simple hypothesis

$$
\mathrm{H}_{0}: \theta=0
$$

against a sequence of complex local alternatives

$$
\mathrm{H}_{n, 1}: \theta=\frac{t}{\sqrt{n}}, \quad 0<t \leq C, \quad C>0,
$$

which is based on independent identically distributed observations $\mathbf{X}_{n}=\left(X_{1}, \ldots, X_{n}\right)$ with Laplace distribution

$$
f(x, \theta)=\frac{1}{2} e^{-|x-\theta|}, \quad x, \theta \in \mathrm{R}^{1} .
$$

For any fixed $n$ and $t \in(0, C]$ we denote by $\beta_{n}^{*}(t)$ the power of the most powerful test of a level $\alpha \in(0,1)$. Such test always exists by the Neyman-Pearson fundamental lemma (see, for instance, [1], p. 183) and is based on the log-likelihood ratio $\Lambda_{n}(t)$

$$
\Lambda_{n}(t)=\sum_{i=1}^{n}\left(\left|X_{i}\right|-\left|X_{i}-t n^{-1 / 2}\right|\right)
$$

Let $\beta_{n}(t)$ be the power of some asymptotically optimal test with the same level $\alpha$, which is based on the statistic

$$
S_{n}(t)=\frac{t}{\sqrt{n}} \sum_{i=1}^{n} \operatorname{sign}\left(X_{i}\right)-\frac{t^{2}}{2} .
$$

Denote by

$$
\Psi_{n}^{*}\left(\Lambda_{n}(t)\right)=\left\{\begin{array}{ll}
0, & \Lambda_{n}(t)<c_{n, t}, \\
\gamma_{n, t}^{*}, & \Lambda_{n}(t)=c_{n, t}, \\
1, & \Lambda_{n}(t)>c_{n, t},
\end{array} \quad \Psi_{n}\left(S_{n}(t)\right)= \begin{cases}0, & S_{n}(t)<\bar{d}_{n, t}, \\
\gamma_{n, t}, & S_{n}(t)=\bar{d}_{n, t}, \\
1, & S_{n}(t)>\bar{d}_{n, t},\end{cases}\right.
$$

the tests of level $\alpha$, respectively, and

$$
\mathrm{E}_{n, 0} \Psi_{n}^{*}\left(\Lambda_{n}(t)\right)=\mathrm{E}_{n, 0} \Psi_{n}\left(S_{n}(t)\right)=\alpha+o\left(\tau_{n}^{2}\right),
$$

where $\tau_{n} \equiv n^{-1 / 4}, \mathrm{E}_{n, 0}$ and $\mathrm{E}_{n, 1}$ are expectations under $\mathrm{H}_{0}$ and $\mathrm{H}_{n, 1}$ (see (1), (2)), respectively. Then

$$
\beta_{n}^{*}(t)=\mathrm{E}_{n, 1} \Psi_{n}^{*}\left(\Lambda_{n}(t)\right), \quad \beta_{n}(t)=\mathrm{E}_{n, 1} \Psi_{n}\left(S_{n}(t)\right) .
$$

We obtained the following results (see [9], theorems 2.1 and 3.3). 
THEOREM 1.1. For the power $\beta_{n}(t)$ uniformly in $t \in[0, C], C \geq 0$, the following asymptotic expansion is valid

$$
\begin{aligned}
\beta_{n}(t)= & \Phi\left(t-u_{\alpha}\right)-\frac{t^{2}}{2 \sqrt{n}} \varphi\left(t-u_{\alpha}\right) \\
& +\frac{t}{2 n} \varphi\left(t-u_{\alpha}\right)\left(\frac{t^{2}}{3}-\frac{t^{3}}{4}\left(t-u_{\alpha}\right)-\frac{1}{6}\left(u_{\alpha}^{2}+u_{\alpha} t-3 t^{2}+24 \delta_{n}\left(1-\delta_{n}\right)-3\right)\right) \\
& +\mathcal{O}\left(n^{-3 / 2}\right),
\end{aligned}
$$

where

$$
\delta_{n}=\frac{n+1}{2}+\frac{\sqrt{n} u_{\alpha}}{2}-\left[\frac{n+1}{2}+\frac{\sqrt{n} u_{\alpha}}{2}\right]+\mathcal{O}\left(n^{-1 / 2}\right),
$$

where $[y]$ is an integer part of $y \in \mathrm{R}^{1}$.

THEOREM 1.2. For the power $\beta_{n}^{*}(t)$ uniformly in $t \in[0, C], C \geq 0$, the following asymptotic expansion is valid

$$
\begin{aligned}
\beta_{n}^{*}(t)= & \Phi\left(t-u_{\alpha}\right)-\frac{t^{2}}{6 \sqrt{n}} \varphi\left(t-u_{\alpha}\right) \\
& -\frac{t}{12 n}\left(\frac{t^{4}}{6}-\frac{t^{2}}{3}-1-u_{\alpha}\left\{\frac{t^{3}}{6}-u_{\alpha}+t\right\}\right) \varphi\left(t-u_{\alpha}\right)+\mathcal{O}\left(n^{-3 / 2}\right),
\end{aligned}
$$

where $\Phi(x), \varphi(x)$ are the standard normal distribution function and the standard normal density function, respectively, $\Phi\left(u_{\alpha}\right)=1-\alpha$.

From the theorems it immediately follows that the difference between the powers has the order $n^{-1 / 2}$ and

$$
r(t) \equiv \lim _{n \rightarrow \infty} \sqrt{n}\left(\beta_{n}^{*}(t)-\beta_{n}(t)\right)=\frac{t^{2}}{3} \varphi\left(u_{\alpha}-t\right) .
$$

2. Formula for the power loss. The proof of the formula (6) is based on asymptotic expansions for the powers $\beta_{n}(t)$ and $\beta_{n}^{*}(t)$ (see previous section). But there is an interest in proving the fact in general form as the theorem 3.2.1 in [1] and the theorem 2.1 in [5] have. The interest is dictated by non-regularity of Laplace distribution and an explicit form of the formula in the theorem 3.2.1 in [1] and the theorem 2.1 in [5]. For simplicity we omit the dependence on parameter $t$ when the latter does not influence reasoning. The explicit form is expressed through a conditional variance of the limit of differences between statistics $S_{n}$ (see (5)) and $\Lambda_{n}$ (see (4)) given the statistic $\Lambda$ as a limit distribution for the sequence $\left\{\Lambda_{n}\right\}$

$$
r=\frac{1}{2} e^{b} \mathrm{D}[\Delta \mid \Lambda=b] p(b),
$$

where $\Delta$ and $\Lambda$ are random variables such that under $H_{0}$ (see (1))

$$
\begin{gathered}
\left(\tau_{n}^{-1} \Delta_{n}, \Lambda_{n}\right) \stackrel{D}{\longrightarrow}(\Delta, \Lambda), \\
\Delta_{n}=S_{n}-\Lambda_{n}, \quad b=\Phi_{1}^{-1}(1-\alpha) .
\end{gathered}
$$

Here $\Phi_{1}(x)$ is the limiting distribution function of $\Lambda_{n}$ under $\mathrm{H}_{0}, p(x)$ is its density function and $\tau_{n}=n^{-1 / 4}$ for the case of Laplace distribution. Formula (7) is useful when 
asymptotic expansions just as in [9] are cumbersome to obtain. In their present form the sufficient conditions of the formula (7) do hold for a variety of statistics of interest, e.g. for rank statistics (R-statistics), linear combinations of order statistics (L-statistics), and U-statistics, also in those cases, where $\Delta_{n}$ does admit a stochastic expansion in terms of sums of independent and identically distributed random variables under some assumptions (see [5]).

All of cases mentioned above have regular properties in contrast to Laplace distribution. Due to this non-regularity the statistic $S_{n}$ (see (5)) has a lattice distribution, and consequently one of regular conditions for the statistic $S_{n}$ does not hold, the analog of Cramér's condition (C) (see [1], theorem 3.2.1, assumption (ii)). Also the difference (6) has order $n^{-1 / 2}$ (see also lemma 3.2 in [10]) in contrast to the order $n^{-1}$ as theorem 2.1 in [5] says. From our results (see lemmas 2.1, 3.1 in [10])

$$
\begin{gathered}
\mathcal{L}\left(\Lambda_{n} \mid \mathrm{H}_{0}\right) \rightarrow \mathcal{N}\left(-\frac{t^{2}}{2}, t^{2}\right), \\
\mathcal{L}\left(\tau_{n}^{-1} \Delta_{n} \mid \mathrm{H}_{0}\right) \rightarrow \mathcal{N}\left(0, \frac{2 t^{3}}{3}\right), \\
\mathcal{L}\left(\left(\tau_{n}^{-1} \Delta_{n}, \Lambda_{n}\right) \mid \mathrm{H}_{0}\right) \rightarrow \mathcal{N}_{2}\left(0, \frac{2 t^{3}}{3}, 0,-\frac{t^{2}}{2}, t^{2}\right),
\end{gathered}
$$

where $\mathcal{N}$ and $\mathcal{N}_{2}$ are univariate and bivariate normal distributions, respectively, we obtain the non-regular stochastic expansion for $\Lambda_{n}$ (see (3.4) in [10])

$$
\Lambda_{n}=S_{n}-\tau_{n}\left(\tau_{n}^{-1} \Delta_{n}\right)=S_{n}-\tau_{n}\left(\tau_{n}^{-1} \sum_{i=1}^{n}\left(-\frac{t^{2}}{2 n}-2\left(X_{i}-\frac{t}{\sqrt{n}}\right) \mathbf{1}_{[0, t / \sqrt{n}]}\left(X_{i}\right)\right)\right),
$$

where $\tau_{n}=n^{-1 / 4}$ in contrast to regular cases when $\tau_{n}=n^{-1 / 2}$.

To prove the validity of the formula (7) for the case of Laplace distribution we consider some properties of $\Lambda_{n}$ and $\Delta_{n}$ (lemmas 2.1-2.3). From lemma 3.2 in [9] (see also [6]) we have

LemMa 2.1. For $\tau_{n}=n^{-1 / 4}$ and continuously differentiable function $\Phi_{1}(x)$ (independent of $n), \Phi_{1}(x)$ having a bounded derivative $p(x)=\Phi_{1}^{\prime}(x)>0$, the following relations are valid

(i)

$$
\sup _{x}\left|\mathrm{P}_{n, 0}\left(\Lambda_{n}<x\right)-\Phi_{1}(x)\right|=\mathcal{O}\left(\tau_{n}^{2}\right)
$$

(ii)

$$
\sup _{x \leq x_{0}} \mathrm{P}_{n, 1}\left(x \leq \Lambda_{n} \leq x+\tau_{n}^{2+\beta}\right)=o\left(\tau_{n}^{2}\right)
$$

for some $\beta>0$ and any $x_{0} \in \mathrm{R}^{1}$,

where $\mathrm{P}_{n, 0}$ and $\mathrm{P}_{n, 1}$ are the distributions of $\mathbf{X}_{n}$ under $\mathrm{H}_{0}$ and $\mathrm{H}_{n, 1}$, respectively.

We proved (see lemma 3.4 in [9]) the following lemma.

LEMma 2.2. For any $x>0$ the following inequality is valid

$$
\mathrm{P}_{n, 0}\left(\tau_{n}^{-1}\left|\Delta_{n}\right| \geq x\right) \leq C e^{-x}, \quad C>0 .
$$


From lemma 2.2 and (8) we have (see lemma 2.2 in [8])

Lemma 2.3. For $\tau_{n}=n^{-1 / 4}$ and $\eta_{n}=n^{-1 / 8}$ the following relations hold

$$
\eta_{n}^{-1} \mathrm{E}_{n, 0} \Delta_{n}^{2} \mathbf{1}_{\left(\eta_{n}, \infty\right)}\left(\left|\Delta_{n}\right|\right)=o\left(\tau_{n}^{2}\right)
$$

$$
\mathrm{E}_{n, 0} e^{\Lambda_{n}} \mathbf{1}_{\left(\eta_{n}, \infty\right)}\left(\left|\Delta_{n}\right|\right)=o\left(\tau_{n}^{2}\right),
$$

where $\mathbf{1}_{A}(\cdot)$ is the indicator of a set $A$.

Lemma 2.1 immediately implies (see lemma 2.4 in [2])

Lemma 2.4. For $\beta_{n}^{*}$ the following relation is valid

$$
\beta_{n}^{*}=\mathrm{P}_{n, 1}\left(\Lambda_{n}>c_{n}\right)+o\left(\tau_{n}^{2}\right) .
$$

We shall consider the following smoothed random variables

$$
\tilde{\Lambda}_{n}=\Lambda_{n}+\xi_{n}, \quad \xi_{n} \sim \mathcal{N}\left(0, \sigma_{n}^{2}\right),
$$

where

$$
\sigma_{n}^{2}=\mathcal{O}\left(\tau_{n}^{4+4 \beta}\right),
$$

$\beta>0$ is a constant as in lemma 2.1, and the random variable $\xi_{n}$ does not depend on $\mathbf{X}_{n}$ under $\mathrm{H}_{0}$. Denote by

$$
\tilde{\beta}_{n}^{*}=\mathrm{E}_{n, 0} e^{\tilde{\Lambda}_{n}} \mathbf{1}_{\left(c_{n}, \infty\right)}\left(\tilde{\Lambda}_{n}\right), \quad \tilde{\beta}_{n}=\mathrm{E}_{n, 0} e^{\tilde{\Lambda}_{n}} \Psi_{n}\left(S_{n}\right)
$$

the smoothed versions of the powers.

Using properties of $\xi_{n}$ (see (11), (12)), lemmas 2.1,2.4, lemma 7.1.2 from [7], and for $X \sim \mathcal{N}\left(0, \sigma^{2}\right)$ and any $a \in \mathrm{R}^{1}$ the relation $\mathrm{E} e^{a X}=e^{\frac{a^{2} \sigma^{2}}{2}}$, we obtained (see lemma 2.6 in $[2])$

Lemma 2.5. For the powers $\beta_{n}$ and $\beta_{n}^{*}$ the following relations hold

$$
\beta_{n}=\tilde{\beta}_{n}+o\left(\tau_{n}^{2}\right), \quad \beta_{n}^{*}=\tilde{\beta}_{n}^{*}+o\left(\tau_{n}^{2}\right) .
$$

In lemma 2.5 we also used the fact that $f(x, \theta)>0$ for all $x, \theta \in \mathrm{R}^{1}$ (see (3)) and the relation $\mathrm{E}_{n, 0} e^{\Lambda_{n}}=1$. The same assumptions are needed to obtain (see lemma 2.7 in [2]) LEMMA 2.6. The following relation is valid

$$
\mathrm{E}_{n, 0} \Psi_{n}^{*}\left(\tilde{\Lambda}_{n}\right)=\mathrm{E}_{n, 0} \mathbf{1}_{\left(c_{n}, \infty\right)}\left(\tilde{\Lambda}_{n}\right)=\alpha+o\left(\tau_{n}^{2}\right) .
$$

From lemmas 2.5, 2.6, 3.5 (see next section) the difference $\beta_{n}^{*}-\beta_{n}$ can be expressed as

$$
\begin{aligned}
\beta_{n}^{*}-\beta_{n} & =\tilde{\beta}_{n}^{*}-\tilde{\beta}_{n}+o\left(\tau_{n}^{2}\right)=\mathrm{E}_{n, 0} e^{\tilde{\Lambda}_{n}}\left(\mathbf{1}_{\left(c_{n}, \infty\right)}\left(\tilde{\Lambda}_{n}\right)-\Psi_{n}\left(S_{n}\right)\right)+o\left(\tau_{n}^{2}\right) \\
& =\mathrm{E}_{n, 0}\left(e^{\tilde{\Lambda}_{n}}-e^{\bar{d}_{n}}\right)\left(\mathbf{1}_{\left(c_{n}, \infty\right)}\left(\tilde{\Lambda}_{n}\right)-\Psi_{n}\left(S_{n}\right)\right)+o\left(\tau_{n}^{2}\right) \equiv \tilde{A}_{n}+\tilde{B}_{n}+o\left(\tau_{n}^{2}\right),
\end{aligned}
$$

where

$$
\begin{aligned}
& \tilde{A}_{n}=\mathrm{E}_{n, 0}\left(e^{\tilde{\Lambda}_{n}}-e^{\bar{d}_{n}}\right)\left(\mathbf{1}_{\left(-\infty, \bar{d}_{n}\right)}\left(\tilde{\Lambda}_{n}\right)-\mathbf{1}_{\left(-\infty, c_{n}\right)}\left(\tilde{\Lambda}_{n}\right)\right), \\
& \tilde{B}_{n}=\mathrm{E}_{n, 0}\left(e^{\tilde{\Lambda}_{n}}-e^{\bar{d}_{n}}\right)\left(1-\Psi_{n}\left(S_{n}\right)-\mathbf{1}_{\left(-\infty, \bar{d}_{n}\right)}\left(\tilde{\Lambda}_{n}\right)\right) .
\end{aligned}
$$


Let

$$
D_{n}=c_{n}-\bar{d}_{n}
$$

Lemmas $3.5-3.7$ of the next section imply that

$$
\begin{gathered}
D_{n}=-\tau_{n} \mathrm{E}[\Delta \mid \Lambda=b]+o\left(\tau_{n}\right), \\
\tilde{A}_{n}=-\frac{1}{2} D_{n}^{2} e^{b} p(b)+o\left(\tau_{n}^{2}\right), \\
\tilde{B}_{n}=\frac{1}{2} \tau_{n}^{2} e^{b} \mathrm{E}\left[\Delta^{2} \mid \Lambda=b\right] p(b)+o\left(\tau_{n}^{2}\right),
\end{gathered}
$$

where $b=\Phi_{1}^{-1}(1-\alpha)$ (see lemma 2.1). From (15)-(17) we have

THEOREM 2.7. For the case of Laplace distribution the following formula is valid

$$
\lim _{n \rightarrow \infty} \tau_{n}^{-2}\left(\beta_{n}^{*}-\beta_{n}\right)=\frac{1}{2} e^{b} \mathrm{D}[\Delta \mid \Lambda=b] p(b) .
$$

3. Additional lemmas. In this section we briefly give the review of the proofs of additional lemmas which imply the results of the previous section. The detailed proofs of the lemmas are in [2].

We shall consider the following functions

$$
\begin{gathered}
\bar{Q}_{n, l}(x)=\int_{|z| \leq \eta_{n} \tau_{n}^{-1}} z^{l}\left[\mathrm{P}_{n, 0}\left(\tau_{n}^{-1} \Delta_{n}<z \mid \tilde{\Lambda}_{n}=x-\tau_{n} z\right)-\mathbf{1}_{(0, \infty)}(z)\right] p_{n}\left(x-\tau_{n} z\right) d z \\
\tilde{Q}_{n, l}(x)=\int_{-\infty}^{\infty} z^{l}\left[\mathrm{P}_{n, 0}\left(\tau_{n}^{-1} \Delta_{n}<z \mid \tilde{\Lambda}_{n}=x-\tau_{n} z\right)-\mathbf{1}_{(0, \infty)}(z)\right] p_{n}\left(x-\tau_{n} z\right) d z
\end{gathered}
$$

where $l=0,1$, and $p_{n}(x)$ is density function of $\tilde{\Lambda}_{n}=\Lambda_{n}+\xi_{n}$ (see (11)).

LEMMA 3.1. The following relations hold

$$
\sup _{x}\left|\bar{Q}_{n, l}(x)-\tilde{Q}_{n, l}(x)\right| \rightarrow 0, \quad l=0,1 .
$$

Proof. Here we mainly use the following inequality

$$
\mathrm{P}(X>x \mid Y) \leq \frac{\mathrm{E}\left[|X|^{l+1} \mathbf{1}_{(x, \infty)}(|X|) \mid Y\right]}{x^{l+1}}, \quad x>0,
$$

and lemma 2.3.

Denote by

$$
\begin{gathered}
\tilde{Q}_{n, u, t}^{*}(B)=\mathrm{P}_{n, 0}\left(\tau_{n}^{-1} \Delta_{n}(t) \in B \mid \tilde{\Lambda}_{n}(t)=u\right) p_{n}(u, t), \\
Q_{u, t}^{*}(B)=\mathrm{P}(\Delta(t) \in B \mid \Lambda(t)=u) p(u, t),
\end{gathered}
$$

measures on $\left(\mathrm{R}^{1}, \mathcal{B}^{1}\right)$ depending on parameters $u \in \mathrm{R}^{1}$ and $t \in(0, C], C>0$. Here we explicitly use dependence on $t$. Denote by

$$
\begin{gathered}
\tilde{q}_{n, u, t}^{*}(s) \equiv \int e^{i s x} \tilde{Q}_{n, u, t}^{*}(d x)=\mathrm{E}_{n, 0}\left[e^{i s \tau_{n}^{-1} \Delta_{n}(t)} \mid \tilde{\Lambda}_{n}(t)=u\right] p_{n}(u, t), \\
q_{u, t}^{*}(s) \equiv \int e^{i s x} Q_{u, t}^{*}(d x)=\mathrm{E}\left[e^{i s \Delta(t)} \mid \Lambda(t)=u\right] p(u, t)
\end{gathered}
$$

their characteristic functions, respectively. 
LEMma 3.2. For any $s \in \mathrm{R}^{1}$ and $n \rightarrow \infty$ the following relation holds

$$
\sup _{0<t \leq C} \sup _{u}\left|\tilde{q}_{n, u, t}^{*}(s)-q_{u, t}^{*}(s)\right| \rightarrow 0 .
$$

Proof. For any $s \in \mathrm{R}^{1}$ and some $\delta>0$ we have

$$
\left|\tilde{q}_{n, u, t}^{*}(s)-q_{u, t}^{*}(s)\right| \leq \int\left|\tilde{q}_{n, t}(s, y)-q_{t}(s, y)\right| d y=\int_{|y|<\delta \sqrt{n}}+\int_{\delta \sqrt{n} \leq|y|<n}+\int_{|y|>n}
$$

where

$$
\begin{aligned}
\tilde{q}_{n, t}(s, y) & \equiv \int e^{i y u} \tilde{q}_{n, u, t}^{*}(s) d u=\mathrm{E}_{n, 0} e^{i s \tau_{n}^{-1} \Delta_{n}(t)+i y \tilde{\Lambda}_{n}(t)} \\
& =\mathrm{E} e^{i y \xi_{n}} \mathrm{E}_{n, 0} e^{i s \tau_{n}^{-1} \Delta_{n}(t)+i y \Lambda_{n}(t)} \\
q_{t}(s, y) & \equiv \int e^{i y u} q_{u, t}^{*}(s) d u=\mathrm{E} e^{i s \Delta(t)+i y \Lambda(t)}
\end{aligned}
$$

Let

$$
\Lambda_{n}(t) \equiv \frac{1}{\sqrt{n}} \sum_{i=1}^{n} h_{n, t}\left(X_{i}\right), \quad \tau_{n}^{-1} \Delta_{n}(t) \equiv \frac{1}{\sqrt{n}} \sum_{i=1}^{n} g_{n, t}\left(X_{i}\right)
$$

where

$$
\begin{aligned}
& h_{n, t}(x)=\sqrt{n}\left(|x|-\left|x-\frac{t}{\sqrt{n}}\right|\right)= \begin{cases}-t, & x<0, \\
2 \sqrt{n} x-t, & 0 \leq x \leq \frac{t}{\sqrt{n}} \\
t, & x>\frac{t}{\sqrt{n}}\end{cases} \\
& g_{n, t}(x)= \begin{cases}-\tau_{n}^{-1} \sqrt{n} \frac{t^{2}}{2 n}, \\
-\tau_{n}^{-1} \sqrt{n} \frac{t^{2}}{2 n}-\tau_{n}^{-1} \sqrt{n} 2\left(x-\frac{t}{\sqrt{n}}\right), & x \notin[0, t / \sqrt{n}],\end{cases}
\end{aligned}
$$

From uniform integrability of $h_{n, t}^{2}(x)$ and $g_{n, t}^{2}(x)$ we obtain the following statement (see lemma 6.3 in [5]): for any $s_{0} \in \mathrm{R}^{1}$ there exist $C_{0}>0, y_{0} \in \mathrm{R}^{1}, n_{0} \in N$ and $\delta>0$ such that for $|y| \leq \delta \sqrt{n}, n \geq n_{0}$

$$
\left|\mathrm{E}_{n, 0} e^{i s_{0} \tau_{n}^{-1} \Delta_{n}(t)+i y \Lambda_{n}(t)}\right| \leq C_{0} \exp \left\{-\frac{1}{4} t^{2}\left(y-y_{0}\right)^{2}\right\} .
$$

For the random variable $h_{n, t}\left(X_{1}\right)$ we have

$$
F_{n, t}(x) \equiv \mathrm{P}_{0}\left\{h_{n, t}\left(X_{1}\right)<x\right\}=\left\{\begin{array}{lc}
0, & x \leq-t, \\
\frac{1}{2}+\int_{-t}^{x} \frac{1}{4 \sqrt{n}} e^{-\frac{u+t}{2 \sqrt{n}}} d u, & -t<x \leq t, \\
1, & x>t .
\end{array}\right.
$$

The latter can be expressed as a mixture of an absolutely continuous distribution $F_{n, t, 1}(x)$ and a discrete distribution $F_{n, t, 2}(x),\left(0<b_{n, t}<1\right)$,

$$
\begin{aligned}
F_{n, t}(x) & =b_{n, t} F_{n, t, 1}(x)+\left(1-b_{n, t}\right) F_{n, t, 2}(x) \\
& =\frac{1-e^{-t / \sqrt{n}}}{2} \int_{-\infty}^{x} f_{n, t, 1}(u) d u+\frac{1+e^{-t / \sqrt{n}}}{2} F_{n, t, 2}(x),
\end{aligned}
$$


where

$$
\begin{gathered}
f_{n, t, 1}(x)= \begin{cases}\frac{2}{1-e^{-t / \sqrt{n}}} \frac{1}{4 \sqrt{n}} e^{-\frac{x+t}{2 \sqrt{n}}}, & x \in[-t, t], \\
0, & x \notin[-t, t],\end{cases} \\
F_{n, t, 2}(x)= \begin{cases}0, & x \leq-t, \\
\frac{1}{1+e^{-t / \sqrt{n}}}, & -t<x \leq t, \\
1, & x>t .\end{cases}
\end{gathered}
$$

While for $x \in[-t, t]$ we have $f_{n, t, 1} \rightarrow \frac{1}{2 t}$ as $n \rightarrow \infty$, and $\sup _{x \in[-t, t]} f_{n, t, 1}(x)<M / 2 t<$ $\infty$, then from the inequality (1.16) in [6] (see [6], p. 421) and from the property of a characteristic function for $0<\delta<\pi / 2 t$ there exists $q=q(\delta)>0$ such that for $|y|>\delta$

$$
\left|\int e^{i y x} F_{n, t, 1}(d x)\right| \leq 1-q .
$$

We consider the following sets

$$
B_{n}=\left\{x \in \mathcal{X}:\left|s_{0} g_{n, t}(x)\right| \leq \frac{q}{2} \sqrt{n}\right\} .
$$

We introduce the following conditional distribution

$$
F_{n, t}\left(x \mid B_{n}\right) \equiv \mathrm{P}_{0}\left(h_{n, t}\left(X_{1}\right)<x \mid B_{n}\right)=\frac{\mathrm{P}_{0}\left(\left\{h_{n, t}\left(X_{1}\right)<x\right\} \cap B_{n}\right)}{\mathrm{P}_{0}\left(B_{n}\right)},
$$

where $\mathrm{P}_{0}\left(B_{n}\right)>0$.

For any $s_{0}, t$ and $q$ there exists $n_{1}$ such that for all $n>n_{1}$ the sets $B_{n}$ coincide with $\mathcal{X}$, and the conditional distributions $F_{n, t}\left(x \mid B_{n}\right)$ coincide with unconditional distributions $F_{n, t}(x)$. Let $n>n_{1}$, then

$$
\begin{aligned}
\left|\mathrm{E}_{0} e^{i \frac{s_{0}}{\sqrt{n}} g_{n, t}\left(X_{1}\right)+i y h_{n, t}\left(X_{1}\right)}\right| & =\left|\mathrm{E}_{0} \mathrm{E}_{0}\left(e^{i \frac{s_{0}}{\sqrt{n}} g_{n, t}\left(X_{1}\right)+i y h_{n, t}\left(X_{1}\right)} \mid h_{n, t}\left(X_{1}\right)=x\right)\right| \\
& =\left|\int e^{i y x} \mathrm{E}_{0}\left(e^{i \frac{s_{0}}{\sqrt{n}} g_{n, t}\left(X_{1}\right)} \mid h_{n, t}\left(X_{1}\right)=x\right) d F_{n, t}(x)\right| .
\end{aligned}
$$

From the definition of $B_{n}$ and the inequality

we have for all $n>n_{1}$

$$
\left|e^{i \frac{s_{0}}{\sqrt{n}} g_{n, t}(x)}-1\right| \leq \frac{\left|s_{0} g_{n, t}(x)\right|}{\sqrt{n}}
$$

$$
\left|\mathrm{E}_{0}\left(e^{i \frac{s_{0}}{\sqrt{n}} y_{n, t}\left(X_{1}\right)} \mid h_{n, t}\left(X_{1}\right)=x\right)-1\right| \leq \frac{q}{2},
$$

which is valid almost surely.

Then we obtain an estimate

$$
\begin{aligned}
& \left|\mathrm{E}_{0} e^{i \frac{s_{0}}{\sqrt{n}} g_{n, t}\left(X_{1}\right)+i y h_{n, t}\left(X_{1}\right)}\right| \\
& \quad \leq b_{n, t}\left|\int e^{i y x} \mathrm{E}_{0}\left(e^{i \frac{s_{0}}{\sqrt{n}} g_{n, t}\left(X_{1}\right)} \mid h_{n, t}\left(X_{1}\right)=x\right) d F_{n, t, 1}(x)\right|+\left(1-b_{n, t}\right) \\
& \quad \leq b_{n, t} \frac{q}{2}+b_{n, t}\left|\int e^{i y x} d F_{n, t, 1}(x)\right|+\left(1-b_{n, t}\right) \leq b_{n, t} \frac{q}{2}+b_{n, t}(1-q)+\left(1-b_{n, t}\right) \\
& \quad \leq \frac{1-e^{-t / \sqrt{n}}}{2}\left(1-\frac{q}{2}\right)+\frac{1+e^{-t / \sqrt{n}}}{2}=1-\frac{C_{1}}{\sqrt{n}}+\mathcal{O}\left(n^{-1}\right),
\end{aligned}
$$

where $C_{1}>0$. 
Then we use (18), (19), lemma 6.3 from [5], lemma 6.3 from [4] and properties of the random variable $\xi_{n}$ (see (11), (12)) to finish the proof.

Let

$$
\begin{aligned}
\tilde{F}_{n, u, t}^{*}(z) & =\mathrm{P}_{n, 0}\left(\tau_{n}^{-1} \Delta_{n}(t)<z \mid \tilde{\Lambda}_{n}(t)=u\right) p_{n}(u, t), \\
F_{u, t}^{*}(z) & =\mathrm{P}(\Delta(t)<z \mid \Lambda(t)=u) p(u, t)
\end{aligned}
$$

be distribution functions depending on parameters $u$ and $t$.

LEMMA 3.3. For any $\varepsilon_{n} \rightarrow 0$ and $n \rightarrow \infty$

$$
\sup _{0<t \leq C} \sup _{u} L\left(\tilde{F}_{n, u+\varepsilon_{n}, t}^{*}, F_{u, t}^{*}\right) \rightarrow 0
$$

where $L\left(F_{1}, F_{2}\right)$ is the Levy distance between distributions.

Proof. From lemma 6.1 in [4], lemma 3.2 and boundedness of $\varepsilon_{n}$ we have

$$
L\left(\tilde{Q}_{n, u+\varepsilon_{n}, t}^{*}, Q_{u, t}^{*}\right) \rightarrow 0
$$

uniformly in $u$ and $t$, because the family $\left\{Q_{u, t}^{*}\right\}$ is tight and bounded in $u$ and $t$ (see lemma 6.2 in [5] and lemma 2.1). Then theorem 2.1 in [3] implies the lemma.

For $l=0,1$ we introduce

$$
Q_{l}(x) \equiv p(x) \int z^{l}\left[\mathrm{P}(\Delta<z \mid \Lambda=x)-\mathbf{1}_{(0, \infty)}(z)\right] d z=-\frac{1}{l+1} \mathrm{E}\left(\Delta^{l+1} \mid \Lambda=x\right) p(x) .
$$

LEMMA 3.4. For $l=0,1$ the following relations hold

$$
\sup _{x}\left|\tilde{Q}_{n, l}(x)-Q_{l}(x)\right| \rightarrow 0 .
$$

Proof. Using lemma 2.2, we obtain the following inequality

$$
\begin{gathered}
\left|\tilde{Q}_{n, l}(x)-Q_{l}(x)\right| \\
\leq\left|\int_{|z| \leq a_{n}} z^{l}\left(\mathrm{P}_{n, 0}\left(\tau_{n}^{-1} \Delta_{n}<z \mid \tilde{\Lambda}_{n}=x-\tau_{n} z\right) p_{n}\left(x-\tau_{n} z\right)-\mathrm{P}(\Delta<z \mid \Lambda=x) p(x)\right) d z\right| \\
+\left|\int_{0}^{a_{n}} z^{l}\left(p_{n}\left(x-\tau_{n} z\right)-p(x)\right) d z\right|+\mathcal{O}\left(e^{-a_{n}}\right),
\end{gathered}
$$

where $a_{n}=\min \left(\lambda_{n}^{-1 / 3}, \kappa_{n}^{-1 / 3}, n^{1 / 8}\right)$, and by lemmas $3.2,3.3$ in [8] and lemma 3.3

$$
\begin{gathered}
\kappa_{n} \equiv \sup _{0<t \leq C} \sup _{x}\left|p_{n}(x)-p(x)\right| \rightarrow 0, \\
\lambda_{n} \equiv \sup _{0<t \leq C} \sup _{x} L\left(\tilde{F}_{n, x-\tau_{n} z, t}^{*}, F_{x, t}^{*}\right) \rightarrow 0 .
\end{gathered}
$$

LEMMA 3.5. For $D_{n}$ the following relation holds

$$
D_{n}=-\tau_{n} \mathrm{E}[\Delta \mid \Lambda=b]+o\left(\tau_{n}\right),
$$

and $\bar{d}_{n} \rightarrow b$.

Proof. Here we mainly use the following relation

$$
F_{\tilde{S}_{n}}(x)=F_{\tilde{\Lambda}_{n}}(x)+\tau_{n} \bar{Q}_{n, 0}(x)+o\left(\tau_{n}^{2}\right),
$$

where $F_{\tilde{\Lambda}_{n}}(x) \equiv \mathrm{P}_{n, 0}\left(\tilde{\Lambda}_{n}<x\right), F_{\tilde{S}_{n}}(x) \equiv \mathrm{P}_{n, 0}\left(S_{n}+\xi_{n}<x\right)$, lemmas $2.1,2.2,3.4$, and relations (8), (10)-(12). 
LEMMA 3.6. For $\tilde{A}_{n}$ the following relation holds

$$
\tilde{A}_{n}=-\frac{1}{2} D_{n}^{2} e^{\bar{d}_{n}} p\left(\bar{d}_{n}\right)+o\left(\tau_{n}^{2}\right) .
$$

Proof. Here we mainly use the following relation (see (13))

$$
\tilde{A}_{n}=\int_{c_{n}}^{\bar{d}_{n}}\left(e^{y}-e^{\bar{d}_{n}}\right) d F_{\tilde{\Lambda}_{n}}(y),
$$

where $F_{\tilde{\Lambda}_{n}}(y) \equiv \mathrm{P}_{n, 0}\left(\tilde{\Lambda}_{n}<y\right)$, integration by parts and lemmas 2.1,3.5.

LEMMA 3.7. For $\tilde{B}_{n}$ the following relation holds

$$
\tilde{B}_{n}=\frac{1}{2} \tau_{n}^{2} e^{\bar{d}_{n}} \mathrm{E}\left[\Delta^{2} \mid \Lambda=\bar{d}_{n}\right] p\left(\bar{d}_{n}\right)+o\left(\tau_{n}^{2}\right) .
$$

Proof. Consider

$$
\tilde{B}_{n}=\mathrm{E}_{n, 0}\left(e^{\tilde{\Lambda}_{n}}-e^{\bar{d}_{n}}\right)\left(\mathbf{1}_{\left(-\infty, \bar{d}_{n}\right)}\left(\tilde{S}_{n}\right)-\mathbf{1}_{\left(-\infty, \bar{d}_{n}\right)}\left(\tilde{\Lambda}_{n}\right)\right)+\rho_{n 1}+\rho_{n 2},
$$

where

$$
\begin{gathered}
\rho_{n 1} \equiv \mathrm{E}_{n, 0}\left(e^{\tilde{\Lambda}_{n}}-e^{\bar{d}_{n}}\right)\left(\mathbf{1}_{\left[\bar{d}_{n}, \infty\right)}\left(S_{n}\right)-\Psi_{n}\left(S_{n}\right)\right)=\left(1-\gamma_{n}\right) \mathrm{E}_{n, 0}\left(e^{\tilde{\Lambda}_{n}}-e^{\bar{d}_{n}}\right) \mathbf{1}_{\left\{\bar{d}_{n}\right\}}\left(S_{n}\right), \\
\rho_{n 2} \equiv \mathrm{E}_{n, 0}\left(e^{\tilde{\Lambda}_{n}}-e^{\bar{d}_{n}}\right)\left(\mathbf{1}_{\left(-\infty, \bar{d}_{n}\right)}\left(S_{n}\right)-\mathbf{1}_{\left(-\infty, \bar{d}_{n}\right)}\left(\tilde{S}_{n}\right)\right) .
\end{gathered}
$$

From lemma 2.2 we obtain (see lemma 3.7 in [2])

$$
\left|\rho_{n 1}\right|=o\left(\tau_{n}^{2}\right), \quad\left|\rho_{n 2}\right|=o\left(\tau_{n}^{2}\right) .
$$

Now we can express $\tilde{B}_{n}$ as

$$
\tilde{B}_{n}=\mathrm{E}_{n, 0}\left(e^{\tilde{\Lambda}_{n}}-e^{\bar{d}_{n}}\right)\left(\mathbf{1}_{\left(-\infty, \bar{d}_{n}\right)}\left(\tilde{S}_{n}\right)-\mathbf{1}_{\left(-\infty, \bar{d}_{n}\right)}\left(\tilde{\Lambda}_{n}\right)\right) \mathbf{1}_{\left[0, \eta_{n}\right)}\left(\left|\Delta_{n}\right|\right)+\rho_{n 3}+o\left(\tau_{n}^{2}\right),
$$

where by lemma 2.2

$$
\left|\rho_{n 3}\right|=o\left(\tau_{n}^{2}\right)
$$

Then for $\eta_{n}=n^{-1 / 8}$

$$
\begin{aligned}
\tilde{B}_{n}= & \tau_{n} e^{\bar{d}_{n}} \int_{|z| \leq \tau_{n}^{-1} \eta_{n}}\left(e^{-\tau_{n} z}-1\right)\left[\mathrm{P}_{n, 0}\left\{\tau_{n}^{-1} \Delta_{n}<z \mid \tilde{\Lambda}_{n}=\bar{d}_{n}-\tau_{n} z\right\}-\mathbf{1}_{(0, \infty)}(z)\right] \\
& \times p_{n}\left(\bar{d}_{n}-\tau_{n} z\right) d z+o\left(\tau_{n}^{2}\right)+\rho_{n 4}
\end{aligned}
$$

where

$$
\begin{aligned}
\left|\rho_{n 4}\right| & \leq 2 \tau_{n} e^{\bar{d}_{n}} \int_{|z| \leq \tau_{n}^{-1} \eta_{n}}\left|e^{-\tau_{n} z}-1\right| \mathrm{P}_{n, 0}\left\{\left|\Delta_{n}\right|>\eta_{n} \mid \tilde{\Lambda}_{n}=\bar{d}_{n}-\tau_{n} z\right\} p_{n}\left(\bar{d}_{n}-\tau_{n} z\right) d z \\
& \leq 2 \eta_{n} e^{\bar{d}_{n}+\eta_{n}} \mathrm{P}_{n, 0}\left\{\left|\Delta_{n}\right|>\eta_{n}\right\}=o\left(\tau_{n}^{2}\right) .
\end{aligned}
$$

From the inequality

$$
\left|e^{-s}-1+s\right| \leq \frac{1}{2} \gamma^{2} e^{\gamma}, \quad|s| \leq \gamma,
$$

where $s=\tau_{n} z$, we can express $\tilde{B}_{n}$ as

$$
\begin{aligned}
\tilde{B}_{n} \equiv & -\tau_{n}^{2} e^{\bar{d}_{n}} \int_{|z| \leq \tau_{n}^{-1} \eta_{n}} z\left[\mathrm{P}_{n, 0}\left\{\tau_{n}^{-1} \Delta_{n}<z \mid \tilde{\Lambda}_{n}=\bar{d}_{n}-\tau_{n} z\right\}-\mathbf{1}_{(0, \infty)}(z)\right] \\
& \times p_{n}\left(\bar{d}_{n}-\tau_{n} z\right) d z+o\left(\tau_{n}^{2}\right)+\rho_{n 5}
\end{aligned}
$$


where

$$
\begin{aligned}
\left|\rho_{n 5}\right| \leq & \frac{1}{2} \tau_{n}^{2} \eta_{n} e^{\bar{d}_{n}+\eta_{n}} \int_{|z| \leq \tau_{n}^{-1} \eta_{n}}|z|\left|\mathrm{P}_{n, 0}\left\{\tau_{n}^{-1} \Delta_{n}<z \mid \tilde{\Lambda}_{n}=\bar{d}_{n}-\tau_{n} z\right\}-\mathbf{1}_{(0, \infty)}(z)\right| \\
& \times p_{n}\left(\bar{d}_{n}-\tau_{n} z\right) d z .
\end{aligned}
$$

The latter integral is equal to $-\bar{Q}_{n, 1}\left(\bar{d}_{n}\right)$, and as follows from the proof of lemma 3.4.4 in $[1]$

$$
\rho_{n 5}=o\left(\tau_{n}^{2}\right)
$$

Then

$$
\tilde{B}_{n}=-\tau_{n}^{2} e^{\bar{d}_{n}} \bar{Q}_{n, 1}\left(\bar{d}_{n}\right)+o\left(\tau_{n}^{2}\right),
$$

and from lemma 3.4 for $l=1$ we obtain

$$
\bar{Q}_{n, 1}\left(\bar{d}_{n}\right) \rightarrow Q_{1}\left(\bar{d}_{n}\right) \equiv-\frac{1}{2} \mathrm{E}\left[\Delta^{2} \mid \Lambda=\bar{d}_{n}\right] p\left(\bar{d}_{n}\right)
$$

4. Conclusions. In this paper we prove theorem 2.7 without an analog of Cramér's (C) condition for the statistic $S_{n}$. Using the theory developed in [4]-[6], we mainly use properties of $\Lambda_{n}$ (see lemma 2.1) and introduce a new condition on $\Delta_{n}$ (see lemma 2.2). Note that the assumptions 2 (ii), (iii) of theorem 3.2.1 in [1] (see lemma 2.3) are not sufficient for theorem 2.7 for the case of Laplace distribution, and we use a more strict condition (lemma 2.2). Also theorem 2.7 was proved in [8] without using lemma 2.2, when we consider the critical value $\bar{d}_{n}$ of the asymptotically optimal test as one of values of the lattice statistic $S_{n}$, that is

$$
\bar{d}_{n}=\min \left\{x: \mathrm{P}_{n, 0}\left\{S_{n} \leq x\right\} \geq 1-\alpha+o\left(\tau_{n}^{2}\right)\right\} .
$$

The latter corresponds to the statement of the problem of testing statistical hypotheses.

\section{References}

[1] V. E. Bening, Asymptotic Theory of Testing Statistical Hypotheses, VSP, Utrecht, 2000.

[2] V. E. Bening and R. A. Korolev, On asymptotic behavior of the powers of the tests for the case of Laplace distribution, Informatics and its Applications, IPI RAN 4 (2010), no. 2, 63-74 (in Russian).

[3] P. Billingsley, Convergence of Probability Measures, Wiley, 1999.

[4] D. M. Chibisov, An asymptotic expansion for distributions of $C(\alpha)$ test statistics, Lecture Notes in Statistics 2, Springer, 1980, 63-96.

[5] D. M. Chibisov, Calculation of the deficiency of asymptotically efficient tests, Teor. Ver. i Primen. 30 (1985), no. 2, 289-310 (in Russian).

[6] D. M. Chibisov and W. R. van Zwet, On the edgeworth expansion for the logarithm of the likelihood ratio. I, Teor. Ver. i Primen. 29 (1984), no. 3, 417-439.

[7] W. Feller, An Introduction to Probability Theory and Its Applications, Vol. 1, 3rd ed., Wiley, New York, 1968.

[8] R. A. Korolev, On the formula for the limit of the normalized difference between the powers of the tests for the case of Laplace distribution, Vestnik Tverskogo gos. univer., ser. Priklad. matematika 9 (2010), no. 1 (16), 5-24 (in Russian). 
[9] R. A. Korolev and V. E. Bening, Asymptotic expansions for power of tests for the case of Laplace distribution, Vestnik Tverskogo gos. univer., ser. Priklad. matematika, 3(10) (2008), 97-107 (in Russian).

[10] R. A. Korolev, A. V. Testova and V. E. Bening, On power of asymptotically optimal test for the case of Laplace distribution, Vestnik Tverskogo gos. univer., ser. Priklad. matematika, 8 (2008), no. 4(64), 5-23 (in Russian).

[11] S. Kotz, T. J. Kozubowski and K. Podgórski, The Laplace Distribution and Generalizations: A Revisit with Applications to Communications, Economics, Engineering, and Finance, Birkhäuser, Boston, 2001. 